\title{
Hybrid control of a hydro-pneumatic tractor suspension
}

\author{
G. Panzani, T. Colombo, S.M. Savaresi and L. Zaccarian
}

\begin{abstract}
We consider the set-point regulation of a non-standard hydro-pneumatic suspension architecture used in commercial tractors, which allows regulating both the stroke and the pressure in the suspension. The model reveals an affine dynamic comprising two single integrators whose actuation is performed by way of suitably switching constant input selections. We design the switching input by way of a hybrid representation, providing necessary and sufficient conditions for the global stabilizability, and proposing two constructive hybrid control laws. The first control law solves the stabilization problem, while the second one can be used to suitably reduce the number of switches of the input, thereby reducing the aging of the actuators. Both control laws are tested in simulation, and assessed in terms of performance and robustness in the presence of parametric uncertainties.
\end{abstract}

\section{INTRODUCTION}

Despite the traditional attention to mechanical topics and issues, agricultural vehicles design has recently started to wink at electronic control systems in order to improve vehicle dynamics performances, see [3]. Besides automatic guidance works, which date back to early noughties (see e.g. [2], [10]), driver comfort is one of the main subject addressed in the recent literature, [8], [9], [13], [14], [16].

Particular attention has been given to vehicle vertical dynamics and vibrations, as typical tractor working scenarios involve uneven soil and irregular roads. Among the several possibilities, the hydropneumatic suspension technology is an interesting suspension one since it allows, with a relatively simple mechanical architecture, to actively produce forces to counteract the effect of road roughness on the sprung masses. Such technology can be beneficially employed on tractors, being capable of sustaining significant loads and exploit already installed hardware (oil-pump and circuits, which are typically present on tractors for the use of implements).

However, when agricultural applications are considered, slower actuation dynamics (w.r.t. the mentioned car counterpart) are to be expected, given the increased volumes and oil flows required. With this limitation, the control objective is usually to centre the suspension position in the available stroke during standard operations, in order to minimize possible collisions with the end-strokes; moreover the suspension can be controlled to set different vehicle chassis geometry and change weights distribution, which can increase the efficiency of specific agricultural processes: such problem is usually called suspension levelling.

Suspension levelling is presented and discussed in the scientific literature. The most common control approach to the problem is presented in [6], [7], [11], [17], [18], where a continuous control action (coming from classical PID or sliding mode controllers) is approximated by means of a PWM actuation of on-off valves, usually flanked by heuristic and fuzzy logic rules to handle uncertainties or application specific issues. In the recent work, [15] a hybrid model of an air-suspension system is proposed and an MPC control strategy is employed to regulate the suspension.

In the present work a non-standard hydro-pneumatic suspension architecture is considered, that allows also to actively change the suspension stiffness by properly regulating the air-spring pressure: thus the control problem becomes multi-objective: the hereby proposed solution lies within the hybrid system framework presented by [5]. Moreover a specific design parameter is also introduced to moderate the number of switches. To overcome the typical implementation and computational burden of MPC

G. Panzani, T. Colombo and S.M. Savaresi are with Dipartimento di Elettronica, Informazione e Bioingegneria, Politecnico di Milano, Italy. email: giulio.panzani@polimi.it

L. Zaccarian is with LAAS-CNRS, Université de Toulouse, CNRS, Toulouse (France) and Dip. di Ingegneria Industriale, University of Trento (Italy). 
solutions, the control law is explicit and it is based on the minimization of a Lyapunov function: this easily allows also to prove the asymptotic stability of the closed-loop system and its robustness.

The proposed modelling framework and the arising control laws well fit within the context of piecewise affine systems, well studied in the literature (see, e.g., the recent survey [12]). While many recent works provide solutions to the corresponding general nonlinear design problem (see, e.g., [1], [4]), a few results allow dealing with the peculiar features of our application, wherein all dynamical matrices involved in the switching system are equal to zero, so that only global asymptotic stability can be obtained (global exponential bounds are not achievable). In addition, the second design solution proposed is associated to the interesting property of reducing the number of switches of the control law, which is somewhat hard to be found in the existing literature. Thus, besides the technological/application-specific advantages, the presented hybrid controller proves to be a valid solution to the broader class of high-order switched affine systems with zero dynamic matrices (namely featuring a simple integral behaviour).

The paper is structured as follows. In Section II we describe the system at stake. In Section III we explain the adopted control architecture and prove its effectiveness. Finally, in Section IV we discuss some simulation results.

\section{PRoblem StATEMENT}

\section{A. Modelling of a hydro-pneumatic suspension}

The considered system is a double acting hydro-pneumatic suspension connected to a feeding hydraulic circuit composed by a tank, a volumetric pump and three on-off valves PISTON , ROD $_{\mathrm{v}}$ and PUMP $_{\mathrm{v}}$ (Fig. 1). The cylinder consists of two chambers, called Rod and Piston, each connected to an
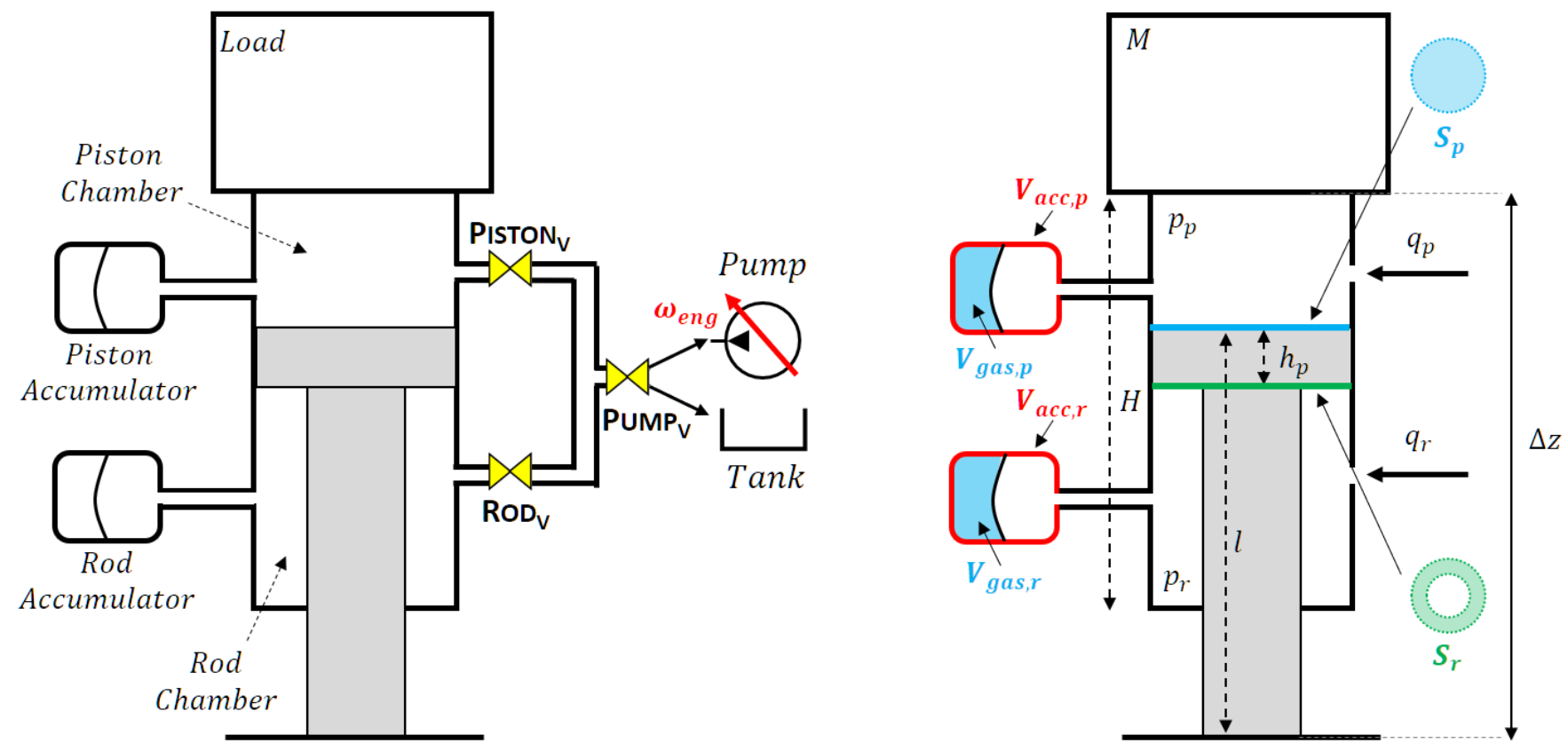

Fig. 1: Hydro-pneumatic suspension layout (left), geometry and symbols (right).

gas spring accumulator; $p_{p}$ and $p_{r}$ are the respective chamber pressures. The hydraulic circuit can be controlled to connect one of the two cylinder's chambers to the pump or to the tank, in order to insert or remove oil from each chamber independently: in this way is possible to modify the suspension height $\Delta z$ and the pressure $p_{r}$ independently.

In order to obtain the suspension dynamics model, the fluid-pressure dynamics are neglected, hence the pressure in each accumulator can be considered equal to the pressure of the respective chamber; gas transformation is considered isothermal i.e. $p V_{g a s, i}=N R T_{i}$, where $T_{i}$ is constant; finally, suspensions 
oscillation dynamics, damping and inertias, are neglected, therefore the suspension position and pressure only depend on the inserted oil volume.

Considering these assumptions, along with the symbols defined in Figure 1, equations (1) can be written.

$$
\begin{aligned}
& \dot{V}_{p}=q_{p} \\
& \dot{V}_{r}=q_{r} \\
& V_{p}+V_{g a s, p}=V_{a c c, p}+S_{p}(\Delta z-l) \\
& V_{r}+V_{g a s, r}=V_{a c c, r}+S_{r}\left(H-\Delta z+l-h_{p}\right) \\
& M g=p_{p} S_{p}-p_{r} S_{r} \\
& p_{p} V_{g a s, p}=N_{p} R T_{p} \\
& p_{r} V_{g a s, r}=N_{r} R T_{r}
\end{aligned}
$$

Equations (1a) represent the oil volume balances for both piston and rod chambers, while equations in (1b) describe the kinematic relations between the suspension position $\Delta z$ and the oil/gas volumes. Equation (1c) is the vertical static force balance and finally equations (1d) are the perfect gas law for the gas in rod and piston accumulators. $V_{p}$ and $V_{r}$ are the overall oil volumes inside both the cylinder chamber and in the respective accumulator (for both piston and rod chambers), while $q_{p}$ and $q_{r}$ are the oil flows provided by the feeding hydraulic circuit. $V_{a c c, i}$ and $V_{g a s, i}$ are the total accumulators volume and the portion occupied by the gas. $S_{p}$ and $S_{r}$ are the effective areas on which the respective chamber pressure acts, while $h_{p}, H$ and $l$ are geometrical cylinder dimensions; finally, $M$ is the mass that burdens on the suspension.

The flows $q_{p}$ and $q_{r}$ depend on how the three valves are controlled; all three valves have two discrete states: the piston valve PISTON ${ }_{\mathrm{v}}$, is the access to the Piston chamber and can be kept open $\left(\right.$ PISTON $_{\mathrm{v}}=1$ ) or closed $\left(\right.$ PISTON $_{\mathrm{v}}=0$ ); the same holds for $\mathrm{ROD}_{\mathrm{v}}$, which is the access to the Rod chamber. Finally the valve $\mathrm{PUMP}_{\mathrm{v}}$, which is the only access to the whole suspension circuit, can be controlled to be connected to the pump $\left(\operatorname{PUMP}_{\mathrm{v}}=1\right)$ or the tank $\left(\mathrm{PUMP}_{\mathrm{v}}=0\right)$, and it cannot be closed. As it is not possible to keep PISTON ${ }_{v}$ and $\operatorname{ROD}_{\mathrm{v}}$ open at the same time and there is no use in keeping them closed having $\operatorname{PUMP}_{\mathrm{v}}=1$, there exist only five control configurations, summarized in the first three columns of Table I.

TABLE I: Discrete valve configurations and corresponding oil flow model.

\begin{tabular}{c|c|c|c|l}
$\mathrm{u}_{\mathrm{th}}$ config & PUMP $_{\mathrm{v}}$ & ROD $_{\mathrm{v}}$ & PISTON $_{\mathrm{v}}$ & Oil flow \\
\hline 0 & 0 & 0 & 0 & $\begin{array}{l}q_{p}=0 \\
q_{r}=0\end{array}$ \\
\hline 1 & 1 & 0 & 1 & $\begin{array}{l}q_{p}=k_{p} \cdot \omega_{\text {eng }} \\
q_{r}=0\end{array}$ \\
\hline 2 & 0 & 1 & 0 & $\begin{array}{l}q_{p}=0 \\
q_{r}=-k_{v_{1}} \sqrt{p_{r}}\end{array}$ \\
\hline 3 & 1 & 1 & 0 & $\begin{array}{l}q_{p}=0 \\
q_{r}=k_{p} \cdot \omega_{e n g}\end{array}$ \\
\hline 4 & 0 & 0 & 1 & $\begin{array}{l}q_{p}=-k_{v_{4}} \sqrt{p_{p}} \\
q_{r}=0\end{array}$ \\
\hline
\end{tabular}

It is possible to derive the physical model for the oil flows for each configuration. It should be highlighted that:

- the pump is a rotational volumetric pump, which is rigidly connected to the tractor engine shaft and for this reason, the suspension incoming oil flow can be modelled as linearly dependent on the engine speed $\omega_{\text {eng }}$; 
- when the oil exits the suspension chambers it follows the typical tank emptying law, where the outgoing flow depends nonlinearly on the difference between the chamber and the ambient pressure (that equals the atmospheric one and can be assumed negligible).

According to the selected configuration, the oil flow can be computed with the expressions listed in the last column of Table I: $k_{p}$ is the linear coefficient between the engine speed and the pump oil flow, while $k_{v_{1}}$ and $k_{v_{4}}$ are the lumped flow factors of the respective control configurations.

Multiplying each equation in (1b) by the respective pressure, substituting (1d) and using the relationship between the rod and the piston pressures imposed by equation (1c), suspension equations (1) can be rewritten as:

$$
\begin{aligned}
& \dot{V}_{p}=q_{p, j}\left(p_{r}, \omega_{\text {eng }}\right) \\
& \dot{V}_{r}=q_{r, j}\left(p_{r}, \omega_{\text {eng }}\right) \\
& \left(p_{r} \frac{S_{r}}{S_{p}}+\frac{M g}{S_{p}}\right) V_{p}+N_{p} R T_{p}=\left(p_{r} \frac{S_{r}}{S_{p}}+\frac{M g}{S_{p}}\right) V_{a c c, p} \\
& +\left(p_{r} \frac{S_{r}}{S_{p}}+\frac{M g}{S_{p}}\right) S_{p}(\Delta z-l) \\
& p_{r} V_{r}+N_{r} R T_{r}=p_{r} V_{a c c, r}+p_{r} S_{r}\left(H-\Delta z+l-h_{p}\right)
\end{aligned}
$$

By defining the state variable vector $v=\left[\begin{array}{ll}V_{p} & V_{r}\end{array}\right]^{T}$, collecting the system outputs in vector $y=$ $\left[\begin{array}{ll}p_{r} & \Delta z\end{array}\right]^{T}$ and all system parameters in vector $\beta$, the suspension dynamic equations can be summarized:

$$
\begin{aligned}
& \dot{v}=f(u, y, \beta) \\
& 0=g(v, y, \beta)
\end{aligned}
$$

The first set of equation in (2) describe the hydro-pneumatic suspension dynamics; the second set of equations implicitly and nonlinearly relate the system state variables with its output.

In order to design the control strategy, a linearised model is considered and to do so the suspension dynamics are expressed in terms of the deviations of the state $w$ and the outputs $z$ from a generic state/output constant value $\tilde{v}, \tilde{y}$, representing the suspension nominal working point. Firstly, the linear relationship between the system states and outputs is derived:

$$
y-\tilde{y}=\frac{\nabla_{x} g(\tilde{v}, \tilde{y})}{\nabla_{y} g(\tilde{v}, \tilde{y})}(v-\tilde{v}) \Rightarrow z=C_{(\tilde{v}, \tilde{y})} w
$$

Similarly the state equations can be linearised, yielding:

$$
\dot{w}=f(u, \tilde{y})+\left[\nabla_{y} f(\tilde{v}, \tilde{y}) \cdot C_{(\tilde{v}, \tilde{y})}\right] w
$$

Considering the technological values of the suspension stroke and the chamber pressures, the following considerations hold. Firstly, the expression provided in (3) is valid only if the Jacobian matrix $\nabla_{y} g(\tilde{v}, \tilde{y})$ is invertible; such condition depends on the state/output values and turns out to be always satisfied. The second remark involves equations (4). When the incoming oil flow configurations ( 1 and 3 ) are active, state equations are already linear and do not depend on the $x$. Conversely, a nonlinear expression arises whenever the chambers are connected to the tank (configurations 2 and 4); however, given the high values of pressures required to sustain the vehicle mass and the consequent small variations from the nominal values that can be accepted, it turns out that the first right-hand-side addendum of (4) is very large w.r.t. the second which can be hence neglected. This means that the suspension dynamics, in all the active configurations where oil flows in or out from the chambers, can be considered as those of a simple integrator. Thus, the only system equilibrium is the one achieved with the null configuration $u=0$, where all valves are locked and thus $q_{p, 0}=q_{r, 0}=0$. From equation (2) it results that any constant value of $\mathrm{x}$ and $\mathrm{y}$, within the mechanical suspension design range, are suitable equilibria for the suspension. 
In conclusion, the control-oriented model of the hydro-pneumatic suspension is described by the following LTI system:

$$
\dot{z}=b_{u}
$$

where, for any value of the control variable $u=\{0,1,2,3,4\}, b_{u}$ is treated as a constant vector:

$$
b_{u}=C_{(\tilde{v}, \tilde{y})} \cdot f(u, \tilde{y}) .
$$

It is worthy to stress that, in fact, $b_{u}$ is not constant since its values depend on the engine speed $\omega_{\text {eng }}$ and the nominal conditions $(\tilde{v}, \tilde{y})$. Robustness, w.r.t. changes of $b_{u}$ is an issue that will be addressed and verified but is guaranteed for small variations by the structural properties of the proposed control architecture (see [5, Ch. 7]).

\section{B. Control design objectives}

There are three main objectives that should be taken into account in the design of a controller for the proposed suspension architecture. The first one is the classical suspension levelling goal, which can be translated into a desired value of $\overline{\Delta z}$; if not controlled, the suspension height can significantly change due to the vertical load variations induced by the road slope or variations of the vehicle mass, which are quite frequent in agricultural tractors by the use of ballast and implements.

The second control objective is strictly related to the suspension architecture under analysis: the possibility of changing the working pressure independently of the suspension height. This degree of freedom opens the possibility to influence the vehicle vertical dynamics, since the suspension stiffness is directly related to the gas pressure. Changing the suspension stiffness can be beneficial in certain driving conditions, e.g. to increase the driver's comfort in case of different road unevenness. From a control perspective, this objective can be translated in a reference value for the rod pressure $\bar{p}_{r}$.

Finally, the third control objective relates to the frequency/number of configuration switches that should be kept limited. On the one side, this helps to increase a higher vale durability; on the other side, excessive valve status changes result in an acoustic discomfort perceived by the tractor driver.

\section{HYBRID CONTROLLER DESIGN}

\section{A. Hybrid control architecture}

The control design problem for plant (5) amounts to the issue of selecting at each instant of time the control variable $u$ within the allowable set $\mathcal{U}:=\{0,1,2,3,4\}$, in such a way that a certain set-point $\bar{z} \in \mathbb{R}^{2}$ is stabilized.

A possible way to represent this control objective is through the use of hybrid dynamics, whose solutions may jump or flow, depending on whether they belong to specific subsets of the state-space. With this modeling framework in mind, we may reconsider the variation of input $u$ as part of the jumping features of solutions (while across jumps the physical state $z$ remains constant), and the variations of the physical states $z$ as part of the flowing features of solutions, where during the flowing phase, input $u$ remains constant. This can be well represented by the suggestive framework of [5] that would lead to the following representation:

$$
\begin{cases}\dot{z}=b_{u} & x=(z, u) \in \mathcal{C} \\ \dot{u}=0, & \\ z^{+}=z & x=(z, u) \in \mathcal{D}, \\ u^{+} \in G(z, u), & \end{cases}
$$

which should be understood as a system where solutions are allowed to flow (according to the differential equation $\dot{x}=\left[\begin{array}{c}b_{u} \\ 0\end{array}\right]$ ) as long as the state $x:=\left[\begin{array}{c}z \\ u\end{array}\right]$ belongs to the so-called flow set $\mathcal{C}$, while solutions are allowed to jump (according to the difference inclusion $x^{+} \in[\underset{G(z, u)}{z}]$ ) as long as the state $x=\left[\begin{array}{l}z \\ u\end{array}\right]$ belongs to the so-called jump set $\mathcal{D}$. 
It is emphasized that representation (7) is associated to an extended state space, therefore the goal of stabilizing dynamics (5) at the reference state $\bar{z}$ can be cast as the goal of selecting they hybrid system data $\mathcal{C}, \mathcal{D}$ and $G$ in such a way that the following compact set:

$$
\mathcal{A}:=\{x=(z, u): u \in \mathcal{U}, z=\bar{z}\}
$$

is globally asymptotically stable.

With this goal in mind, it becomes quite natural to select the flow set $\mathcal{C}$ as the complement of the jump set $\mathcal{D}$, in such a way that solutions will either be able to flow or jump. In addition, to ensure well posedness of the hybrid dynamics (see [5, Ch. 5-6]) we also include the boundary in that selection:

$$
\mathcal{C}:=\overline{\left(\mathbb{R}^{2} \times \mathcal{U}\right) \backslash \mathcal{D}}
$$

so that $\mathcal{C}$ is actually the closed complement of $\mathcal{D}$.

Summarizing, the available degrees of freedom in the hybrid control design are represented by the selection of $\mathcal{D}$, encoding the information about "when" the input $u$ should switch to a new value, and the selection of the jump map $G$, encoding the information about "where" the new value of the input $u$ should be selected. In the next sections we will illustrate two Lyapunov-based ideas for the selection of $\mathcal{D}$ and $G$, both ensuring global asymptotic stability of set $\mathcal{A}$ in (8), and the second one addressing the third objective listed in Section II-B.

\section{B. Solution to the stabilization problem}

In this section we propose Lyapunov-based selections of the jump set $\mathcal{D}$ and of the jump map $G$ in (7) ensuring global asymptotic stability (GAS) of the attractor $\mathcal{A}$. Note that ensuring GAS of this set resolves the first two items of the problem statement in Section II-B. Indeed, one can select the two components of the reference state $\bar{z}$ as the desired levelling goal $\overline{\Delta z}$ and the desired rod pressure $\bar{p}_{r}$. The proposed approach is more general than the specific setting and applies to dynamics of the form (5) for a generic dimension of the state space and of the input space. We will then consider generically $z \in \mathbb{R}^{n}$ and $\mathcal{U}=\{0,1, \ldots, N\}$.

To ensure controllability of the dynamics (5), we make the following assumption, which can be proven to be necessary for globally stabilizing a generic point $\bar{z}$ in $\mathbb{R}^{n}$.

Assumption 1: The set of vectors $b_{i}, i=0, \ldots, N$ form a positive basis of $\mathbb{R}^{n}$. Namely, for each $z \in \mathbb{R}^{n}$, there exist non-negative numbers $\lambda_{i} \geq 0, i=0, \ldots, N$, such that $z=\sum_{i=0}^{N} \lambda_{i} b_{i}$.

The necessity of Assumption 1 is immediate as one realizes that failing to satisfy this assumption implies that from each initial conditions $z(0)$, there is at least a half space that cannot be reached with any time-varying selection of the input $u$. With this assumption in place, we can prove the following lemma, that provides a main ingredient for our Lyapunov-based construction.

Lemma 1: If Assumption 1 holds, then given any selection of $P=P^{T}>0$, there exists a small enough number $\bar{\eta}>0$ such that

$$
\min _{i=0, \ldots, N} z^{T} P b_{i} \leq-\bar{\eta}|z|, \quad \forall z \in \mathbb{R}^{n} .
$$

Based on the result of Lemma 1, we can propose a first choice of $G$ and $\mathcal{D}$ in (7), guaranteeing the first two items of the problem statement in Section II-B, but without a clear attention towards the number of switches (item 3). The control law is parametric and depends on an arbitrary matrix $P=P^{T}>0$, whose selection may be dictated by specific performance needs. Indeed, the quadratic function $x^{T} P x$ is guaranteed to be non-decreasing along the arising closed loop. Given any $P=P^{T}>0$, the proposed selection is:

$$
\begin{aligned}
\mathcal{D} & := \\
G(z, u) & :=\underset{u^{+} \in \mathcal{U}}{\operatorname{argmin}}(z-\bar{z})^{T} P b_{u^{+}}
\end{aligned}
$$

where $\eta<\bar{\eta}$ is a design parameter (typically selected small to reduce the switching frequency). The following theorem establishes the desirable closed-loop properties induced by solution (7), (9), (11). 
Theorem 1: Given any value of $P=P^{T}>0$, if Assumption 1 holds, then the attractor $\mathcal{A}$ in (8) is globally asymptotically stable for the closed-loop system (7), (9), (11).

\section{Enhanced solution for reduced switching}

While the control scheme proposed in the previous section is successful at stabilizing the set-point $\bar{z}$, its transient evolution may lead to frequent switching, and this may lead to undesirable usage of the actuators. Due to this reason, we propose here a dynamic extension with the goal of performing some kind of preview $u_{*} \in \mathcal{U}$ of the subsequent value of the input, and essentially encoding the feature that the switch to a new input is delayed if this leads to an improvement in terms of transient perspective.

The modified controller is still based on a quadratic Lyapunov function that in this case depends on the controller state $u_{*} \in \mathcal{U}$ as follows

$$
\begin{aligned}
W\left(e, u_{*}\right) & :=e^{T} P_{u_{*}} e \\
& :=\alpha e^{T} P e+(1-\alpha) e^{T}\left(I-\frac{b_{u_{*}} b_{u_{*}}^{T}}{b_{u_{*}}^{T} b_{u_{*}}}\right) e,
\end{aligned}
$$

where $\alpha \in(0,1]$ is a tuning parameter. For $\alpha=1$, we come back to the previous solution, whereas for $\alpha$ increasingly smaller, we favor the new solution involving the preview action of the controller.

The dynamics of the hybrid controller is not much different from the previous one, and is associated to two jump sets. The first set corresponds to triggering a jump of input $u$ coinciding with the one defined in (11) (except for replacing matrix $P$ with the new state-dependent Lyapunov matrix $P_{u_{*}}$ ):

$$
\mathcal{D}_{u}:=\left\{\left(e, u, u_{*}\right): e^{T} P_{u_{*}} b_{u} \geq-\eta|e|\right\},
$$

where, similar to before, $\eta$ is a tuning parameter that should be selected small, ${ }^{1}$ and which is associated to the jump map

$$
G_{u}\left(e, u_{*}\right):=\underset{u^{+} \in \mathcal{U}}{\operatorname{argmin}} e^{T} P_{u_{*}} b_{u^{+}}
$$

The second set corresponds to triggering a jump of state $u_{*}$ and is given by

$$
\begin{aligned}
\mathcal{D}_{u_{*}}:= & \left\{\left(e, u, u_{*}\right):\right. \\
& \left.\min _{u^{+}: e^{T} P b_{u^{+}} \leq 0} W\left(e, u^{+}\right) \leq W\left(e, u_{*}\right)-\varepsilon|e|^{2}\right\},
\end{aligned}
$$

where $\varepsilon>0$ is a design parameter (typically selected small to avoid restricting too much the updates of $u_{*}$ ), which is associated to the jump map

$$
G_{u_{*}}(e):=\underset{u^{+}: e^{T} P b_{u^{+}} \leq 0}{\operatorname{argmin}} W\left(e, u^{+}\right),
$$

With these sets at hand, we select the overall jump set as the union of the two jump sets and the flow set as its closed complement: ${ }^{2}$

$$
\mathcal{D}:=\mathcal{D}_{u_{*}} \cup \mathcal{D}_{u}, \quad \mathcal{C}:=\overline{\left(\mathbb{R}^{n} \times \mathcal{U} \times \mathcal{U}\right) \backslash \mathcal{D}},
$$

which clearly prioritizes jumps over flows. In particular, one could equivalently write the flow set as:

$$
\begin{aligned}
\mathcal{C}=\left\{\left(e, u, u_{*}\right): e^{T} P_{u_{*}} b_{u} \leq-\eta|e|\right. \text { and } \\
\left.\min _{u^{+}: e^{T} P b_{u^{+}} \geq 0} W\left(e, u^{+}\right) \leq W\left(e, u_{*}\right)-\varepsilon|e|^{2}\right\}
\end{aligned}
$$

\footnotetext{
${ }^{1}$ We avoid giving precise definition of the suggested size of $\eta$, whose selection should satisfy the bound assumed in (??), because $\eta$ should be selected as a very small number, in practical tuning.

${ }^{2}$ To avoid overloading the notation, we use the same symbols as in (7) for the jump and the flow sets, even though we make different selections here.
} 
Remark 1: It is worth to provide an illustration of the rationale behind the mechanism relying on the dynamic augmentation effected by the new state $u_{*}$. We do this for the planar case $z \in \mathbb{R}^{2}$, even though our proof of stability below applies to the general case. First of all, notice that the logic of the new controller coincides with the one introduced in Section III-B when $\alpha=1$. Indeed in that case we have $W=V, P_{u_{*}}=P$, and the quantity $u_{*}$ plays no role in the selection of $u$. The extreme and opposite case is the one where $\alpha=0$ (which is not feasible, but should be considered as a limiting case with very small values of $\alpha$ ). In that case, one sees that $P_{u^{*}} \approx\left(I-\frac{b_{u_{*}} b_{u_{*}}^{T}}{b_{u_{*}}^{T} b_{u_{*}}}\right)$ is a projection on the orthogonal subspace $\mathcal{U}_{*}^{\perp}$ to the one $\mathcal{U}_{*}$ generated by $u_{*}$ and that the jump rule governing $u_{*}$ ensures that it is selected as the direction associated to the smallest value of that projection (up to a tolerance $\varepsilon$ inserted to avoid undesired chattering). Coming back to the logic behind the variation of $u$ (and $P_{u^{*}}$ ), we obtain that the control scheme first selects a value of $u$ that drives to zero the error component on the orthogonal subspace $\mathcal{U}_{*}^{\perp}$ (because along the constant direction $b_{u}$ the gradient of $W$ preserves its sign until that subspace is reached) and then, once the subspace $\mathcal{U}_{*}$ generated by $u_{*}$ is reached, $W$ is almost zero and $u$ must switch (necessarily to $u_{*}$ because otherwise $W$ would start growing again). That last switch brings the error to zero along $u_{*}$. Clearly, this "binary" behavior ensures that only one switch occurs, so that the error is brought to zero with minimum switching. For any value of $\alpha$ between 0 and 1 , the expected transient is an intermediate one among the two above mentioned extreme cases. It is worth mentioning that the solution cannot work for $\alpha=0$, because this would lead to a non-positive definite matrix $P_{u^{*}}$, and then we cannot use Lemma 1 to apply the LaSalle invariance principle. Indeed, in that case solutions remain bounded, but there are nonconverging constant solutions.

To ensure an outer semicontinuous selection of the jump map (which enables the use of La Salle's invariance principle in our proof), the overall jump map is then selected as a function whose graph is the union of the graphs of the two functions $G_{u}$ and $G_{u_{*}}$. Denoting $x=\left(e, u, u_{*}\right)$, it can be written as:

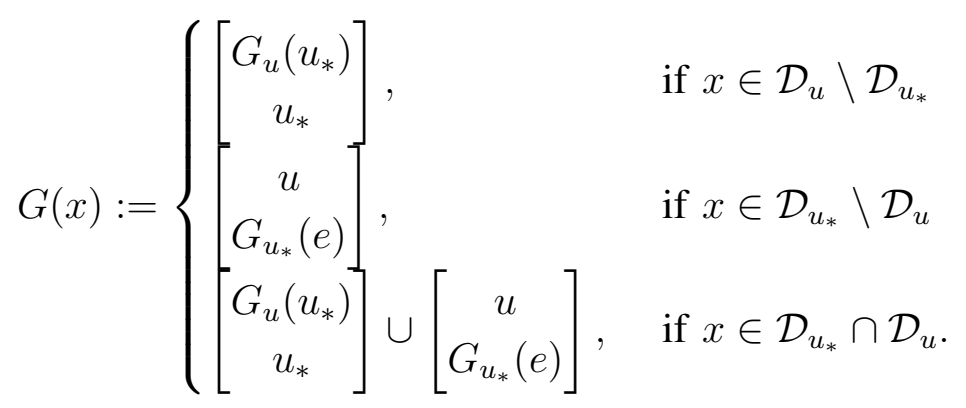

The overall scheme can be represented by generalizing the hybrid dynamics (7) to the case where the controller has an internal state $u_{*}$, corresponding to:

$$
\begin{cases}\dot{z}=b_{u} & \\
\dot{u}=0, & x \in \mathcal{C} \\
\dot{u}_{*}=0 & \\
z^{+}=z & \\
{\left[\begin{array}{c}
u^{+} \\
\left(u_{*}\right)^{+}
\end{array}\right] \in G(x),} & x \in \mathcal{D} .\end{cases}
$$

The next theorem parallels the results of Theorem 1 for this enhanced dynamical control scheme.

Theorem 2: Given any value of $P=P^{T}>0$, if Assumption 1 holds, then the attractor

$$
\mathcal{A}:=\left\{\left(z, u, u_{*}\right): z=\bar{z}, u \in \mathcal{U}, u_{*} \in \mathcal{U}\right\},
$$

is globally asymptotically stable for the closed-loop (20). 


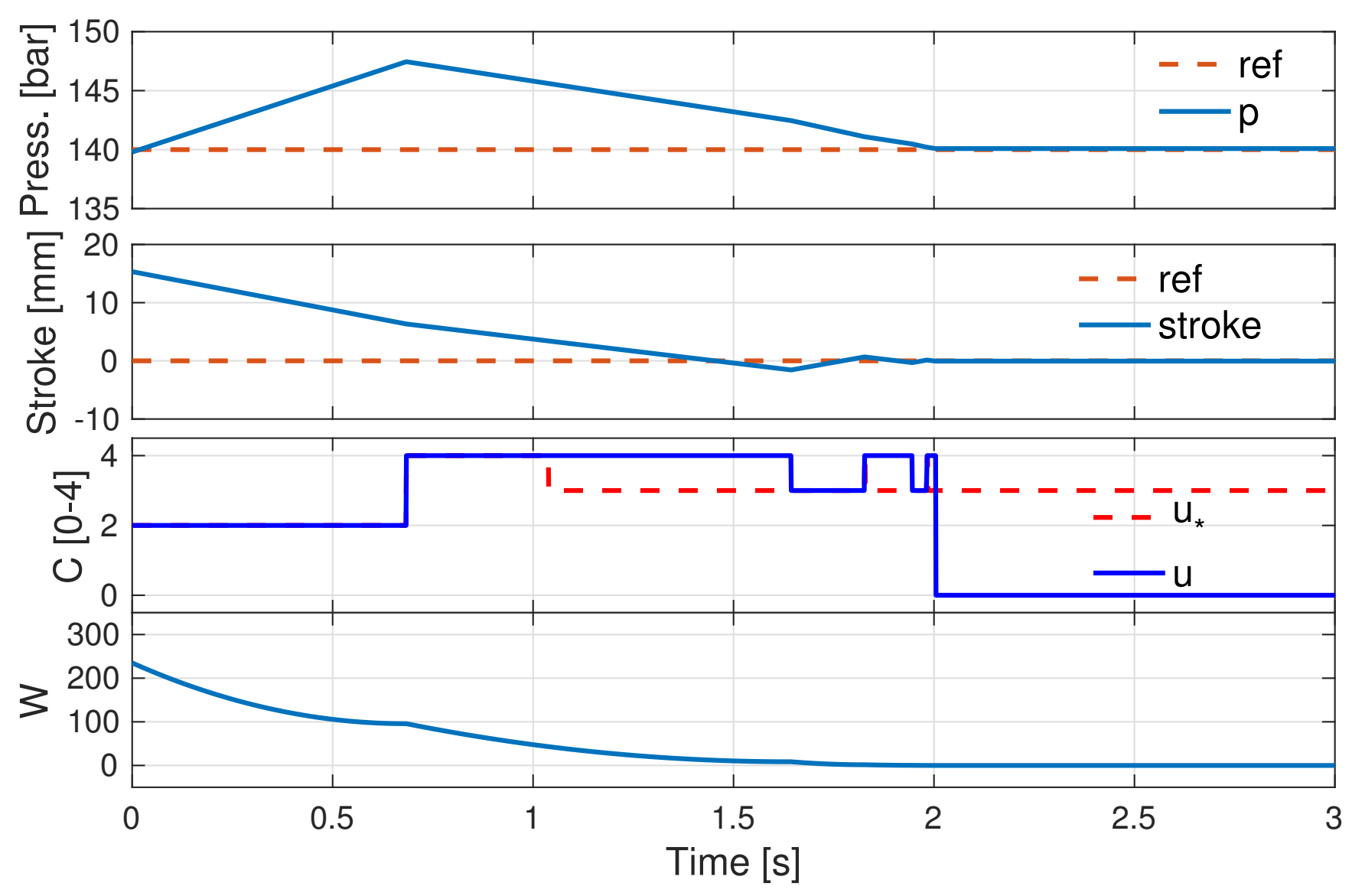

Fig. 2: Closed-loop behavior with $P=I$ and $\alpha=1$.

\section{Simulations}

\section{A. Nominal case}

The control solution proposed in Section III-C is applied to the control of the considered suspension. Results are shown in terms of variables y, rather than the deviations z. In Figure 2 the time evolution of $\mathbf{y}$ and $u, u_{*}$ are shown, along with the values of the Lyapunov function $W$ in (12). In this simulation the nominal behaviour of the system is depicted, i.e. the matrix $P$ is set equal to the identity and the parameter $\alpha$ is set equal to 1 ; notice that with this choice of $\alpha, u_{*}$ has no role in the choice of the actuated configuration and the control law reduces to the one proposed in Section III-B. Given that for the configuration proposed Assumption 1 holds, as a results the controller is capable of driving the pressure and the suspension height (stroke) to the desired values. In all our simulations, we only use input values in the set $\{1,2,3,4\}$ for the controller implementation, and we define an additional local strategy that activates mode $\{0\}$ (corresponding to $b_{0}=\left[\begin{array}{l}0 \\ 0\end{array}\right]$ ), in a small ball around the reference state $\bar{z}$, having size $\varepsilon=10^{-2}$. We also introduce a hysteresis switching mechanism reactivating the global control logic using the four active modes, whenever the error $z-\bar{z}$ is larger in norm that $2 \cdot 10^{-2}$, even though this mechanism is never activated in the presented simulations.

The effect of different choices of $P$, in the definition of the Lyapunov function can be appreciated in Figure 3 where the state trajectories for two different choices of $P$ are shown. In particular:

$$
P_{s}=\left[\begin{array}{cc}
0.25 & 0 \\
0 & 1
\end{array}\right], \quad P_{p}=\left[\begin{array}{cc}
1 & 0 \\
0 & 0.25
\end{array}\right]
$$

The arising simulations are consistent with the choices of $P$ : when $P_{s}$ is used, the controller drives the system towards the desired reference values following a trajectory that prioritizes reducing the error on the suspension stroke. Conversely when $P_{p}$ is considered, the system drives rapidly the state towards zero pressure errors, and then the whole error state towards the reference. 


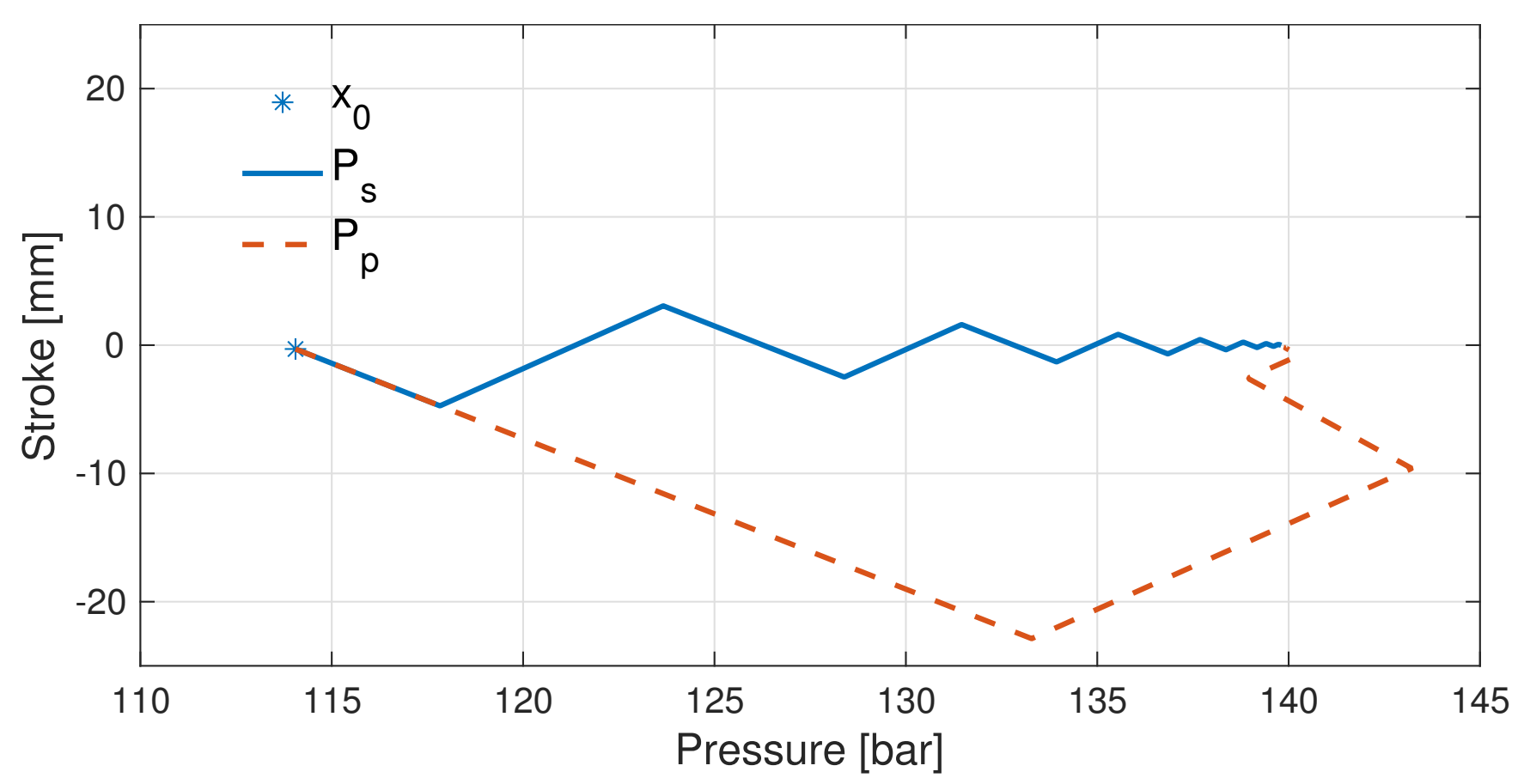

Fig. 3: State trajectories for different tunings of matrix $P$, with $\alpha=1$.

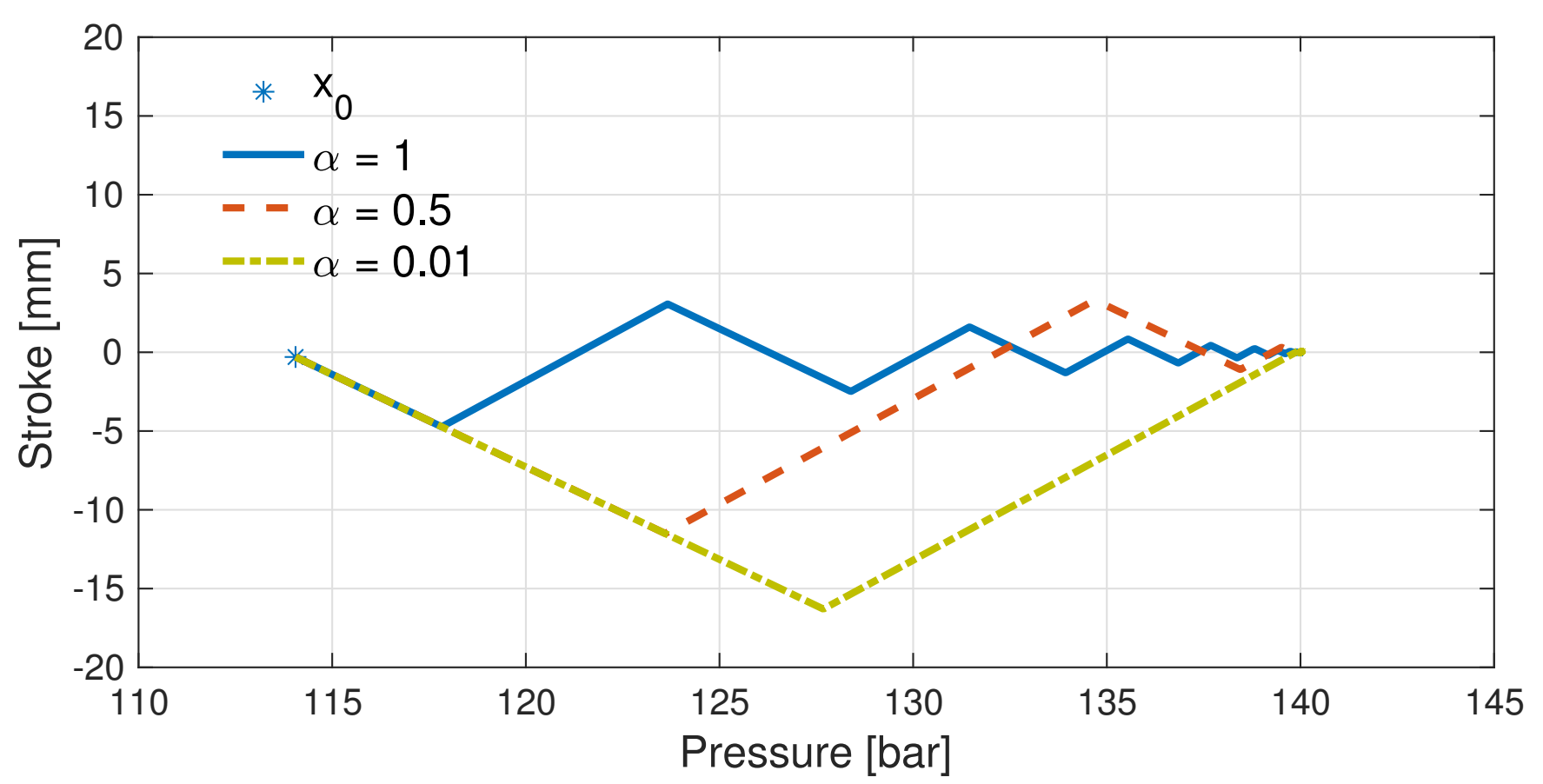

Fig. 4: State trajectories for different tunings of $\alpha$.

\section{B. Sensitivity to parameter $\alpha$}

In order to show the effect of different choices of $\alpha$, the situation of Figure 3 for $P=P_{s}$ is considered. In the nominal case $\alpha=1$, the control input performs several switches: in order to reduce their number (without changing $P$ ), as discussed in Section III-C and Remark 1, the parameter $\alpha$ is decreased. The simulated results are shown in Figure 4. It can be seen how this parameter is actually effective in reducing the switch numbers: when $\alpha$ decreases towards zero the limit situation of a single switch is achieved. It should be stressed that there is not a unique value of $\alpha$ that induces a single switch 
trajectory but it can vary for different initial conditions $x_{0}$.

\section{Robustness to uncertainties}

Since the proposed controller design and the established global asymptotic stability results are based on the framework proposed in [5], the stability properties of the closed loop are indeed robust to sufficiently small perturbations (according to the general robustness properties established in [5, Ch. 7]). These robustness properties have been tested in the presence of parametric uncertainties. The following scenario has been considered: firstly, an increase of $30 \%$ of the engine speed which, according to the model in Table I, produces an increased size of the vectors of input selections $u=1$ and $u=3$. Additionally, a random uncertainty on the remaining vectors has been considered. A pictorial comparison between the nominal (used in the control law (20)) and the perturbed (used as system parameters during the simulation) vectors $b_{u}$ is shown in Figure 5a. The nominal and perturbed closed-loop responses are shown in Figure $5 \mathrm{~b}$ and 6 . It can be seen that the closed-loop system proves robustness of stability in the presence of model uncertainties, and it is capable of driving the states towards the setpoint values. Given that configurations $u=1$ and $u=3$ produce a faster system response, in the perturbed scenario the system reaches the steady-state slightly before the nominal case. Clearly, the state trajectories of Figure $5 \mathrm{~b}$ become different only when configurations $u=2$ and $u=4$ are selected.

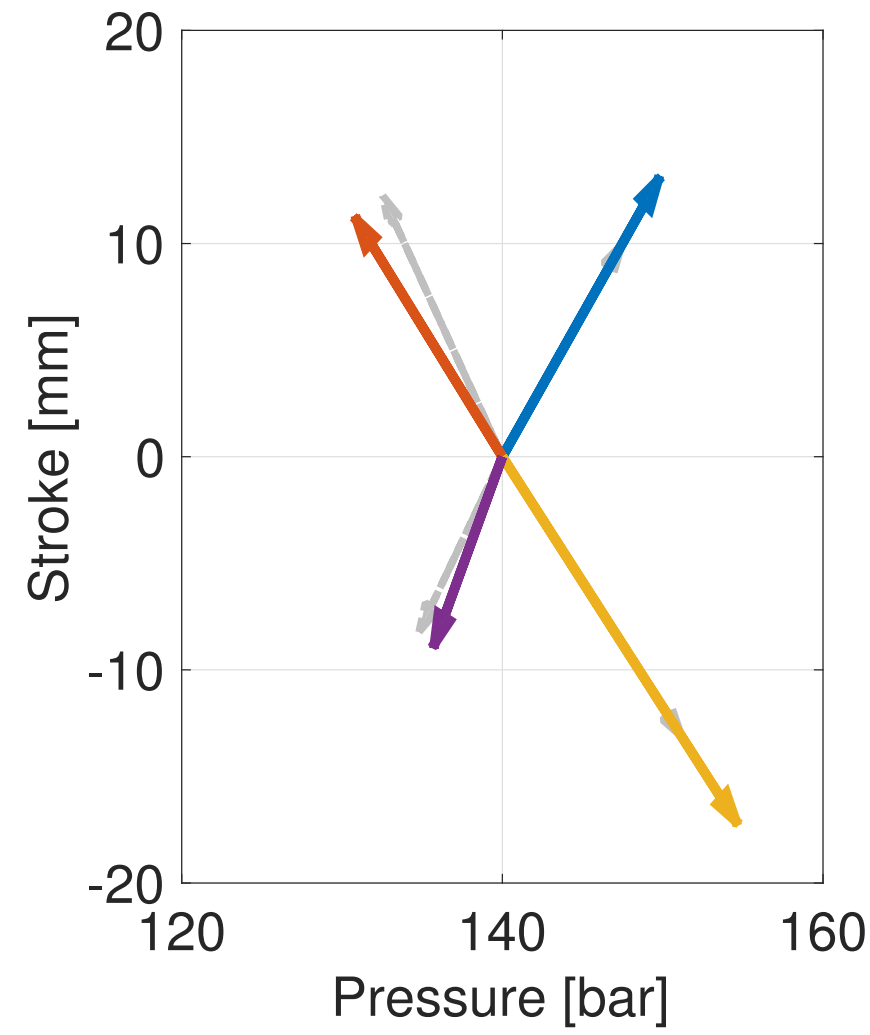

(a) Configurations.

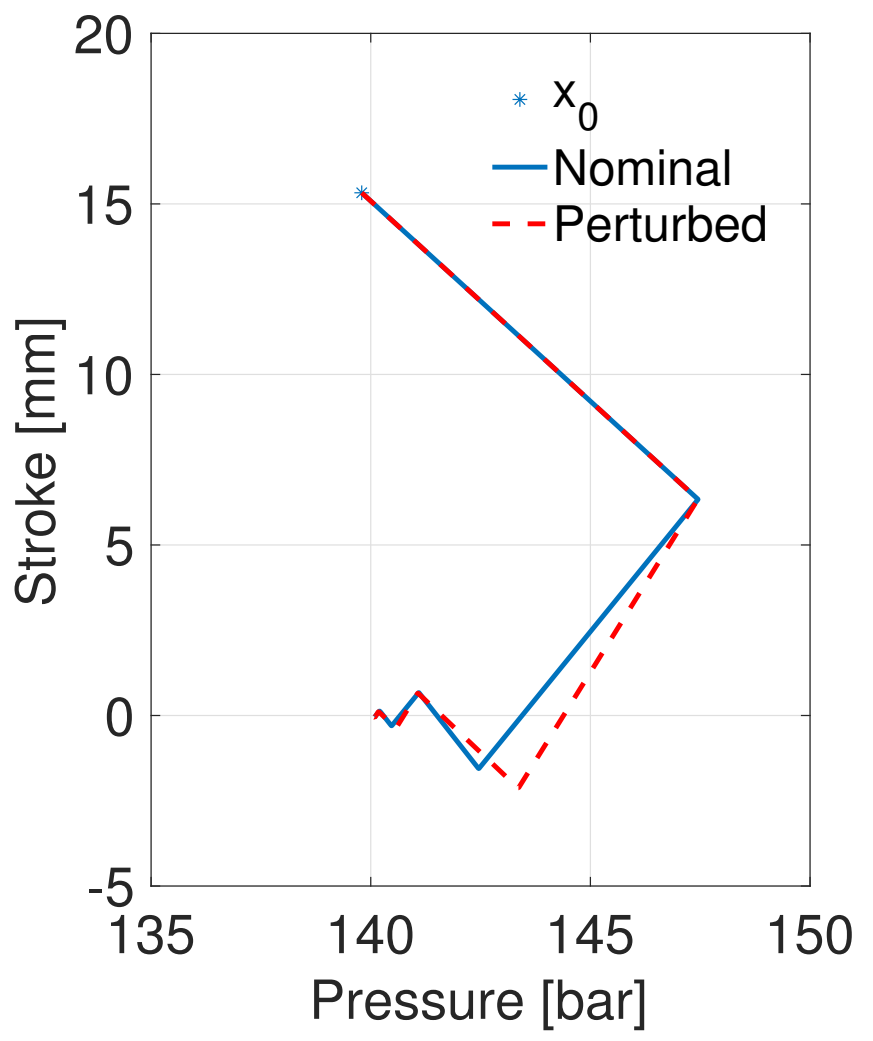

(b) Phase portrait.

Fig. 5: Comparison between the nominal (light gray) and the perturbed (thick colored) system configurations, 5a. Comparison between the state trajectories of the nominal (solid) system and the perturbed (dahsed) one, $5 b$.

\section{CONCLUSIONS}

In this paper we have addressed the control problem for a hydro-pneumatic tractor suspension where both the pressure and the stroke can be independently regulated. The derived model corresponds to a 

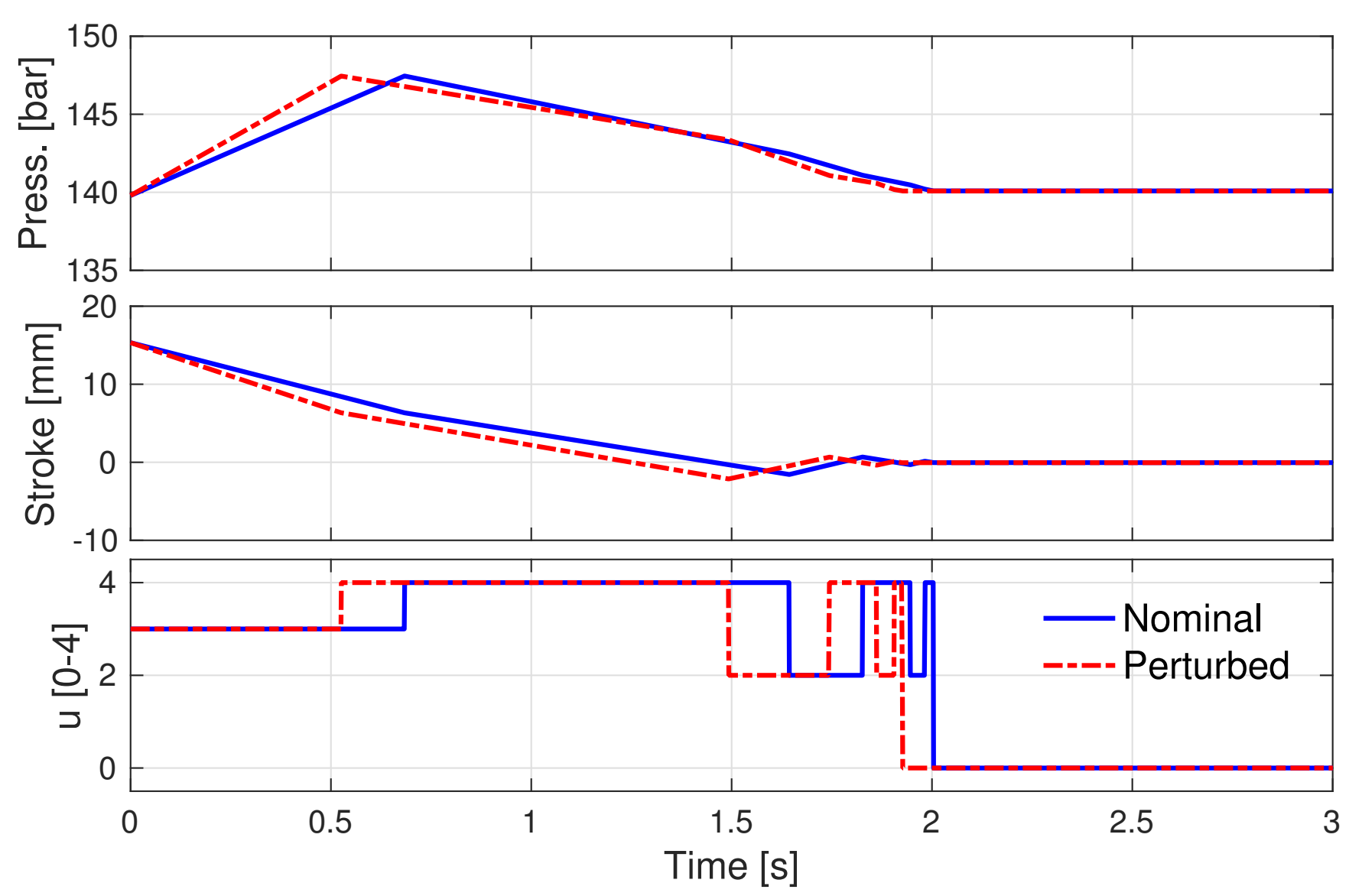

Fig. 6: Comparison between the state trajectories of the nominal (solid) system and the perturbed (dahsed) one.

switching affine system wherein the control input amounts to selecting the active mode, and can be selected among a number of available modes (five total modes). To suitably represent such a switching dynamics, the control design problem has been cast as a hybrid control design problem and two solutions have been proposed, both ensuring global asymptotic stability (GAS) of the set-point configuration, under some necessary (and sufficient) assumptions on the system parameters. In addition to achieving GAS, we have shown that our second control law is also effective at reducing the total number of switches by appropriately keeping track of the future input value via an additional controller state. All the established results have been tested in simulation. Possible future work includes extending the proposed preview mechanism to the case of larger state-space dimensions, testing the proposed approach on experimental tests, and proposing design techniques with robustness guarantees for large parameter variations.

\section{REFERENCES}

[1] C. Albea-Sanchez, G. Garcia, and L. Zaccarian. Hybrid dynamic modeling and control of switched affine systems: Application to DC-DC converters. In IEEE Conference on Decision and Control, pages 2264-2269, Osaka, Japan, December 2015.

[2] T. Bell. Automatic tractor guidance using carrier-phase differential gps. Computers and electronics in agriculture, 25(1):53-66, 2000.

[3] Eugenio Cavallo, Ester Ferrari, and Mario Coccia. Likely technological trajectories in agricultural tractors by analysing innovative attitudes of farmers. International Journal of Technology, Policy and Management, 15(2):158-177, 2015.

[4] G.S. Deaecto, J.C. Geromel, F.S. Garcia, and J.A. Pomilio. Switched affine systems control design with application to dc-dc converters. IET control theory \& applications, 4(7):1201-1210, 2010.

[5] R. Goebel, R.G. Sanfelice, and A.R. Teel. Hybrid Dynamical Systems: modeling, stability, and robustness. Princeton University Press, 2012.

[6] H. Kim and H. Lee. Height and leveling control of automotive air suspension system using sliding mode approach. IEEE Transactions on Vehicular Technology, 60(5):2027-2041, 2011. 
[7] H. Kim, H. Lee, and H. Kim. Asynchronous and synchronous load leveling compensation algorithm in airspring suspension. In International Conference on Control, Automation and Systems (ICCAS), pages 367-372, 2007.

[8] V. Manzoni, M. Tanelli, S. M. Savaresi, and F. Taroni. Automatic end-of-line tuning for a motion inverter in agricultural tractors. Control Engineering Practice, 18(11):1342-1355, 2010.

[9] G. Panzani, M. Tanelli, S. M. Savaresi, C. Pirola, G. Gavina, and F. Taroni. Transmission control for power-shift agricultural tractors. In American Control Conference, pages 2188-2193, 2010.

[10] John F Reid, Qin Zhang, Noboru Noguchi, and Monte Dickson. Agricultural automatic guidance research in north america. Computers and electronics in agriculture, 25(1):155-167, 2000.

[11] X. Shen, J. Zhang, E. J. Barth, and M. Goldfarb. Nonlinear model-based control of pulse width modulated pneumatic servo systems. Journal of Dynamic Systems, Measurement, and Control, 128(3):663-669, 2006.

[12] R. Shorten, F. Wirth, O. Mason, K. Wulff, and C. King. Stability criteria for switched and hybrid systems. SIAM review, 49(4):545592, 2007.

[13] Kyuhyun Sim, Hwayoung Lee, Ji Won Yoon, Chanho Choi, and Sung-Ho Hwang. Effectiveness evaluation of hydro-pneumatic and semi-active cab suspension for the improvement of ride comfort of agricultural tractors. Journal of Terramechanics, 69:23-32, 2017.

[14] C. Spelta, F. Previdi, S. M. Savaresi, D. Delvecchio, and S. Tremolada. Semi-active control of cab suspension in an agricultural tractor via magneto-rheological actuator. In IEEE International Conference on Control and Automation (ICCA), pages 812-817, 2011.

[15] X. Sun, Y. Cai, L. Chen, Y. Liu, and S. Wang. Vehicle height and posture control of the electronic air suspension system using the hybrid system approach. Vehicle System Dynamics, 54(3):328-352, 2016.

[16] M. Tanelli, G. Panzani, S. M. Savaresi, and C. Pirola. Transmission control for power-shift agricultural tractors: Design and endof-line automatic tuning. Mechatronics, 21(1):285-297, 2011.

[17] C. Yuexia, C. Long, W. Ruochen, X. Xing, S. Yujie, and L. Yanling. Modeling and test on height adjustment system of electricallycontrolled air suspension for agricultural vehicles. International Journal of Agricultural and Biological Engineering, 9(2):40, 2016.

[18] J. Zhao, P. K. Wong, Z. Xie, C. Wei, and F. He. Integrated variable speed-fuzzy pwm control for ride height adjustment of active air suspension systems. In American Control Conference, pages 5700-5705, 2015. 